\title{
A conservative Fourier pseudo-spectral method for the nonlinear Schrödinger equation
}

\author{
Yuezheng Gong ${ }^{1}$, Qi Wang ${ }^{1,2}$, Yushun Wang ${ }^{3 *}$, Jiaxiang Cai ${ }^{4}$ \\ ${ }^{1}$ Beijing Computational Science Research Center, Beijing 100193, China. \\ ${ }^{2}$ Department of Mathematics, IMI and the NonoCenter at USC, \\ University of South Carolina, Columbia, SC 29208, USA. \\ ${ }^{3}$ Jiangsu Provincial Key Laboratory for NSLSCS, School of Mathematical Sciences, \\ Nanjing Normal University, Nanjing 210023, China. \\ ${ }^{4}$ School of Mathematics Science, Huaiyin Normal University, Huaian 223300, China.
}

\begin{abstract}
A Fourier pseudo-spectral method that conserves mass and energy is developed for a two-dimensional nonlinear Schrödinger equation. By establishing the equivalence between the semi-norm in the Fourier pseudo-spectral method and that in the finite difference method, we are able to extend the result in Ref. [56] to prove that the optimal rate of convergence of the new method is in the order of $O\left(N^{-r}+\tau^{2}\right)$ in the discrete $L^{2}$ norm without any restrictions on the grid ratio, where $N$ is the number of modes used in the spectral method and $\tau$ is the time step size. A fast solver is then applied to the discrete nonlinear equation system to speed up the numerical computation for the high order method. Numerical examples are presented to show the efficiency and accuracy of the new method.

Keywords: Nonlinear Schrödinger equation, Fourier pseudo-spectral method, Unconditional convergence, FFT.
\end{abstract}

Mathematics Subject Classification: 65M06, 65M12, 65M70.

\section{Introduction}

The nonlinear Schrödinger (NLS) equation is the most important equation in quantum mechanics [37]. It describes a wide range of physical phenomena [38, 44,45,51], including plasma physics, nonlinear optics, self-focusing in laser pulses, propagation of heat pulses in crystals, and dynamics of Bose-Einstein condensates at the extremely low temperature. In this paper, we consider the following cubic NLS equation in two dimensional space:

$$
i u_{t}+\Delta u+\beta|u|^{2} u=0,(x, y) \in \mathbb{R} \times \mathbb{R}, 0<t \leq T,
$$

subject to the $\left(l_{1}, l_{2}\right)$-periodic boundary condition

$$
u(x, y, t)=u\left(x+l_{1}, y, t\right), u(x, y, t)=u\left(x, y+l_{2}, t\right),(x, y) \in \mathbb{R} \times \mathbb{R}, 0<t \leq T,
$$

and the initial condition

$$
u(x, y, 0)=\varphi(x, y),(x, y) \in \mathbb{R} \times \mathbb{R}
$$

${ }^{*}$ Corresponding author.

E-mail:wangyushun@njnu.edu.cn. 
where $\Delta=\partial_{x x}+\partial_{y y}$ is the 2-dimensional Laplacian operator, $\beta \neq 0$ is a given real constant, $\varphi(x, y)$ is a given $\left(l_{1}, l_{2}\right)$-periodic complex-valued function. The NLS equation (1.1) is focusing for $\beta>0$, and defocusing for $\beta<0$, which is a generic model for the slowly varying envelop of a wave-train in conservative, dispersive, mildly nonlinear wave phenomena. It is also obtained as the subsonic limit of the Zakharov model for Langmuir waves in plasma physics [59]. It has been shown that it is possible for solutions of the two-dimensional NLS equation to develop singularities in finite time [33].

The NLS equation has been studied extensively. For the derivation, well-posedness and dynamical properties of the NLS equation, readers are referred to $[7,18,51]$ and the references therein. In fact, the solution of the initial-periodic boundary value problem (1.1)-(1.3) satisfies the following mass and energy conservation laws

$$
Q(t):=\int_{\Omega}|u(x, y, t)|^{2} d x d y \equiv Q(0)
$$

and

$$
E(t):=\int_{\Omega}\left[|\nabla u(x, y, t)|^{2}-\frac{\beta}{2}|u(x, y, t)|^{4}\right] d x d y \equiv E(0),
$$

where $|\nabla u|^{2}=\left|u_{x}\right|^{2}+\left|u_{y}\right|^{2}$ and $\Omega=\left[0, l_{1}\right] \times\left[0, l_{2}\right]$. These conservation laws imply that, $\|u(\cdot, \cdot, t)\|_{H_{p}^{1}}<\infty$ for the defocusing case or for the focusing case with $\|\varphi\|^{2} \leq \frac{2-\widetilde{\varepsilon}}{4(1+\varepsilon) \beta}$ where $\varepsilon$ and $\widetilde{\varepsilon}$ are two positive numbers which can be arbitrary small. The theory on existence of global solutions of the NLS equation $[18,51]$ implies that, if $\|u(\cdot, \cdot, t)\|_{H_{p}^{1}}<\infty, u(x, y, t) \in$ $L^{\infty}\left(\mathbb{R}^{+}, H_{p}^{1}(\Omega)\right)$.

Numerically, various algorithms have been developed for solving the NLS equation, including the finite difference method $[3,19,23,28,31,35,50,55-57,60]$, compact boundary value methods [27], finite element method [1,2,32,39], discontinuous Galerkin method [40,58], Runge-Kutta or Crank-Nicolson pseudo-spectral method [17,20,29,30,48], time-splitting pseudo-spectral method $[5,6,8,53]$, meshless method [25, 26] and radial basis function collocation method [24], etc. Some comparisons among the various numerical methods for the NLS equation can be found in $[4,19,43,54]$ and the references therein.

For the NLS equation in one dimension, error estimates of different numerical methods have been established. For time-splitting methods, we refer to $[9,22,42,46,53]$ for details. For the implicit Runge-Kutta finite element method, we refer to $[2,47]$ for more details. Analyses on unconditional convergence of the conservative finite difference (CFD) method for the NLS equation in one dimension were conducted in $[19,55]$. In fact, their proofs for CFD scheme rely heavily on not only the discrete conservative property but also the discrete version of the Sobolev inequality in one dimension

$$
\|f\|_{L^{\infty}} \leq C\|f\|_{H^{1}}, \quad \forall f \in H_{0}^{1}(\Omega) \text { or } f \in H_{p}^{1}(\Omega) \text { with } \Omega \subset \mathbb{R},
$$

which immediately implies an a priori uniform bound for $\|f\|_{L^{\infty}}$. However, the extension of the discrete version of the above Sobolev inequality is no longer valid in two dimensions. Thus the techniques used in $[19,55]$ cannot be extended to high dimensions without significant modifications. Due to the difficulty in obtaining an a priori, uniform estimate for the numerical solution, few error estimates are available in the literature for the NLS equation in two dimensions. Gao and Xie [31] proposed a fourth-order alternating direction implicit compact finite difference scheme for a two-dimensional Schrödinger equation, and also used induction argument to prove that their scheme is conditionally convergent in the discrete $L^{2}$ norm. In the analysis, they imposed a strict restriction on the grid ratio. In [56], Wang et al. developed a new technique to analyze a compact finite difference scheme for the NLS equation in two dimensions. They proved 
that their method is convergent in the order of $O\left(h^{4}+\tau^{2}\right)$ in the discrete $L^{2}$ norm without any restrictions on the mesh ratio.

The spectral method is a classical, high order and widely used technique to solve differential equations, both theoretically and numerically [49]. Bridges and Reich [10] first introduced the idea of Fourier spectral discretization to construct multi-symplectic integrator for Hamiltonian systems. Based on their theory, Chen and Qin [20] proposed a multi-symplectic Fourier pseudospectral method for Hamiltonian PDEs and applied it to integrate the NLS equation with periodic boundary conditions. Later, different structure-preserving Fourier pseudo-spectral methods were developed $[12,21,34,35,41]$. However, few convergent results of the Fourier pseudo-spectral method are available. Recently, Cai et al. [13-15] developed and analyzed different structurepreserving Fourier pseudo-spectral methods for the 3D Maxwell's equation. But unconditionally convergent results on Fourier pseudo-spectral method for nonlinear PDEs have not been obtained. For nonlinear problems, once an a priori estimate of the numerical solution in discrete $L^{\infty}$ norm is known, it seems that the error analysis can be carried out using the standard linear theory for evolutionary equations. However, it is hard to be substantiated. Therefore, there has not been a single proof on the unconditional error estimate of the Fourier pseudo-spectral method for nonlinear NLSs. In this paper, we develop a conservative Fourier pseudo-spectral method for solving the initial-periodic boundary value problem of the NLS equation in two dimensions. We show that the semi-norm in the Fourier pseudo-spectral method is equivalent to that in the finite difference method. Once this important connection is established, we prove the method is unconditionally convergent with the order of $O\left(N^{-r}+\tau^{2}\right)$ in the discrete $L^{2}$ norm, using the techniques developed in Ref. [56]. A fast solver is then applied to solve the discrete nonlinear equation system. We present some numerical examples in the end and demonstrate the effectiveness of the new high order method.

The rest of the paper is organized as follows. In Section 2, we define some notations to be used in the proof and present the conservative Fourier pseudo-spectral algorithm for the 2D NLS equation. In Section 3, we prove the existence of the numerical solution by the Browder fixed point theorem. Then, we show the numerical scheme satisfies discrete conservation laws and obtain an a priori estimate. In Section 4, the convergence property of the new scheme is analyzed. Numerical examples are presented to show the efficiency and accuracy of the method in Section 5. Finally, we give a concluding remark in Section 6.

\section{Fourier pseudo-spectral method}

We develop a numerical method to solve the initial-periodic boundary value problem (1.1)-(1.3) in a finite domain $\Omega \times[0, T]$. First, we introduce some notations. For two positive even integers $N_{x}$ and $N_{y}$, we define two step sizes in space: $h_{1}=l_{1} / N_{x}, h_{2}=l_{2} / N_{y}$. Then, the spatial grid points are defined as follows: $\Omega_{h}=\left\{\left(x_{j}, y_{k}\right) \mid j=0,1, \ldots, N_{x}-1 ; k=0,1, \ldots, N_{y}-1\right\}$, where $x_{j}=j h_{1}, 0 \leq j \leq N_{x}-1, y_{k}=k h_{2}, 0 \leq k \leq N_{y}-1$. For a positive integer $N_{t}$, we define the time-step: $\tau=T / N_{t}$. The grid points in space and time are given by $\Omega_{h}^{\tau}=\Omega_{h} \times \Omega_{\tau}$, where $\Omega_{\tau}=\left\{t_{n} \mid t_{n}=n \tau, n=0,1, \ldots, N_{t}\right\}$. Given a grid function $u=\left\{u_{j, k}^{n} \mid\left(x_{j}, y_{k}, t_{n}\right) \in \Omega_{h}^{\tau}\right\}$, we denote

$$
\delta_{x}^{+} u_{j, k}^{n}=\frac{u_{j+1, k}^{n}-u_{j, k}^{n}}{h_{1}}, \delta_{y}^{+} u_{j, k}^{n}=\frac{u_{j, k+1}^{n}-u_{j, k}^{n}}{h_{2}}, \delta_{t}^{+} u_{j, k}^{n}=\frac{u_{j, k}^{n+1}-u_{j, k}^{n}}{\tau}, u_{j, k}^{n+1 / 2}=\frac{u_{j, k}^{n}+u_{j, k}^{n+1}}{2} .
$$

Let $\mathbb{V}_{h}=\left\{u \mid u=\left(u_{j, k}\right),\left(x_{j}, y_{k}\right) \in \Omega_{h}\right\}$ be the space of grid functions defined on $\Omega_{h}$. For any 
two grid functions $u, v \in \mathbb{V}_{h}$, we define the discrete inner product as follows:

$$
(u, v)_{h}=h_{1} h_{2} \sum_{j=0}^{N_{x}-1} \sum_{k=0}^{N_{y}-1} u_{j, k} \bar{v}_{j, k}
$$

The discrete $L^{2}$ norm of $v$ and its difference quotients are defined, respectively, as

$\|v\|_{h}=\sqrt{(v, v)_{h}},\left\|\delta_{x}^{+} v\right\|_{h}=\sqrt{\left(\delta_{x}^{+} v, \delta_{x}^{+} v\right)_{h}},\left\|\delta_{y}^{+} v\right\|_{h}=\sqrt{\left(\delta_{y}^{+} v, \delta_{y}^{+} v\right)_{h}},|v|_{h, 1}=\sqrt{\left\|\delta_{x}^{+} v\right\|_{h}^{2}+\left\|\delta_{y}^{+} v\right\|_{h}^{2}}$.

We also define the discrete $L^{p}(1 \leq p<\infty)$ norm as

$$
\|v\|_{h, p}=\left(h_{1} h_{2} \sum_{j=0}^{N_{x}-1} \sum_{k=0}^{N_{y}-1}\left|v_{j, k}\right|^{p}\right)^{1 / p}
$$

and the discrete $L^{\infty}$ norm as

$$
\|v\|_{h, \infty}=\max _{\left(x_{j}, y_{k}\right) \in \Omega_{h}}\left|v_{j, k}\right| .
$$

We note that the discrete norm $\left\|\delta_{x}^{+} v\right\|_{h},\left\|\delta_{y}^{+} v\right\|_{h},|v|_{h, 1}$ defined above are semi-norms.

For simplicity, we denote $u_{j, k}^{n}=u\left(x_{j}, y_{k}, t_{n}\right)$ and $U_{j, k}^{n}$ as the exact value of $u(x, y, t)$ and its numerical approximation at $\left(x_{j}, y_{k}, t_{n}\right)$, respectively.

\subsection{Fourier pseudo-spectral approximation of spatial derivatives}

We define

$$
S_{N}^{\prime \prime}=\operatorname{span}\left\{g_{j}(x) g_{k}(y), j=0,1, \ldots, N_{x}-1 ; k=0,1, \ldots, N_{y}-1\right\}
$$

as the interpolation space, where $g_{j}(x)$ and $g_{k}(y)$ are trigonometric polynomials of degree $N_{x} / 2$ and $N_{y} / 2$, given respectively by

$$
g_{j}(x)=\frac{1}{N_{x}} \sum_{p=-N_{x} / 2}^{N_{x} / 2} \frac{1}{a_{p}} e^{i p \mu_{1}\left(x-x_{j}\right)}, \quad g_{k}(y)=\frac{1}{N_{y}} \sum_{q=-N_{y} / 2}^{N_{y} / 2} \frac{1}{b_{q}} e^{i q \mu_{2}\left(y-y_{k}\right)},
$$

where $a_{p}=\left\{\begin{array}{l}1,|p|<N_{x} / 2, \\ 2,|p|=N_{x} / 2,\end{array} \quad \mu_{1}=2 \pi / l_{1}, b_{q}=\left\{\begin{array}{l}1,|q|<N_{y} / 2, \\ 2,|q|=N_{y} / 2,\end{array} \quad \mu_{2}=2 \pi / l_{2}\right.\right.$.

We define the interpolation operator $I_{N}: L^{2}(\Omega) \rightarrow S_{N}^{\prime \prime}$ as follows:

$$
I_{N} u(x, y)=\sum_{j=0}^{N_{x}-1} \sum_{k=0}^{N_{y}-1} u_{j, k} g_{j}(x) g_{k}(y)
$$

where $u_{j, k}=u\left(x_{j}, y_{k}\right), g_{j}\left(x_{l}\right)=\delta_{l}^{j}, g_{k}\left(y_{m}\right)=\delta_{m}^{k}$.

To obtain derivative $\partial_{x}^{s_{1}} \partial_{y}^{s_{2}} I_{N} u(x, y)$ at the collocation points $\left(x_{j}, y_{k}\right)$, we differentiate $(2.2)$ and evaluate the resulting expressions at the points $\left(x_{j}, y_{k}\right)$ :

$$
\partial_{x}^{s_{1}} \partial_{y}^{s_{2}} I_{N} u\left(x_{j}, y_{k}\right)=\sum_{l=0}^{N_{x}-1} \sum_{m=0}^{N_{y}-1} u_{l, m} \frac{d^{s_{1}} g_{l}\left(x_{j}\right)}{d x^{s_{1}}} \frac{d^{s_{2}} g_{m}\left(y_{k}\right)}{d y^{s_{2}}}=\left(D_{s_{1}}^{x} \mathbf{u}\left(D_{s_{2}}^{y}\right)^{T}\right)_{j, k}
$$


where $D_{s_{1}}^{x}$ is an $N_{x} \times N_{x}$ matrix and $D_{s_{2}}^{y}$ an $N_{y} \times N_{y}$ matrix, respectively, with elements given by

$$
\left(D_{s_{1}}^{x}\right)_{j, l}=\frac{d^{s_{1}} g_{l}\left(x_{j}\right)}{d x^{s_{1}}}, \quad\left(D_{s_{2}}^{y}\right)_{k, m}=\frac{d^{s_{2}} g_{m}\left(y_{k}\right)}{d y^{s_{2}}},
$$

and $\mathbf{u}=\left(u_{j, k}\right)$ is an $N_{x} \times N_{y}$ matrix. Note that $D_{s}^{x}, D_{s}^{y}$ are real antisymmetric matrices when $s$ is odd; $D_{s}^{x}, D_{s}^{y}$ are real symmetric matrices when $s$ is even. More details can be found in [20,34]. For second derivatives, we have

$$
\partial_{x x} I_{N} u\left(x_{j}, y_{k}\right)=\left(D_{2}^{x} \mathbf{u}\right)_{j, k}, \quad \partial_{y y} I_{N} u\left(x_{j}, y_{k}\right)=\left(\mathbf{u} D_{2}^{y}\right)_{j, k},
$$

where symmetry of $D_{2}^{y}$ is used.

\subsection{Conservative Fourier pseudo-spectral method}

We discretize the NLS equation (1.1)-(1.3) using the Fourier pseudo-spectral method in space and the Crank-Nicolson method in time to arrive at a full-discrete system:

$$
i \delta_{t}^{+} U_{j, k}^{n}+\left(D_{2}^{x} U^{n+1 / 2}\right)_{j, k}+\left(U^{n+1 / 2} D_{2}^{y}\right)_{j, k}+\frac{\beta}{2}\left(\left|U_{j, k}^{n}\right|^{2}+\left|U_{j, k}^{n+1}\right|^{2}\right) U_{j, k}^{n+1 / 2}=0, \quad U^{n} \in \mathbb{V}_{h},
$$

where $j=0,1, \ldots, N_{x}-1, k=0,1, \ldots, N_{y}-1$. For convenience, scheme (2.4) can be written in a matrix form

$$
i \delta_{t}^{+} U^{n}+D_{2}^{x} U^{n+1 / 2}+U^{n+1 / 2} D_{2}^{y}+F\left(U^{n}, U^{n+1}\right)=0, \quad U^{n} \in \mathbb{V}_{h},
$$

where $U^{n}=\left(U_{j k}^{n}\right), F\left(U^{n}, U^{n+1}\right)=\left(F\left(U_{j, k}^{n}, U_{j, k}^{n+1}\right)\right)=\left(\frac{\beta}{4}\left(\left|U_{j, k}^{n}\right|^{2}+\left|U_{j, k}^{n+1}\right|^{2}\right)\left(U_{j, k}^{n}+U_{j, k}^{n+1}\right)\right)$. Here, we define a new semi-norm:

$$
|v|_{h}=\sqrt{\left(-D_{2}^{x} v, v\right)_{h}+\left(-v D_{2}^{y}, v\right)_{h}}, \quad v \in \mathbb{V}_{h} .
$$

Next, we show that $|\cdot|_{h}$ is equivalent to $|\cdot|_{h, 1}$. Thus, definition (2.6) is meaningful.

A matrix in the form of

$$
A=\left(\begin{array}{cccc}
a_{0} & a_{1} & \cdots & a_{n-1} \\
a_{n-1} & a_{0} & \cdots & a_{n-2} \\
\vdots & \vdots & \ddots & \vdots \\
a_{1} & a_{2} & \cdots & a_{0}
\end{array}\right)
$$

is called a circulant matrix [36]. Because the matrix $A$ is determined by the entries in the first row, the matrix can be denoted as

$$
A=C\left(a_{0}, a_{1}, \ldots, a_{n-1}\right) .
$$

According to summation-by-parts formula

$$
\left(\delta_{\alpha}^{+} f, g\right)_{h}+\left(f, \delta_{\alpha}^{-} g\right)_{h}=0, \alpha=x \text { or } y,
$$

we have

$$
\begin{aligned}
\left\|\delta_{x}^{+} u\right\|_{h}^{2} & =-\left(\delta_{x}^{-} \delta_{x}^{+} u, u\right)_{h}=\left(-A_{1} u, u\right)_{h} \\
\left\|\delta_{y}^{+} u\right\|_{h}^{2} & =-\left(\delta_{y}^{-} \delta_{y}^{+} u, u\right)_{h}=\left(-u A_{2}, u\right)_{h}
\end{aligned}
$$

where

$$
A_{1}=\frac{1}{h_{1}^{2}} C(\underbrace{-2,1,0, \ldots, 0,1}_{N_{x}}), \quad A_{2}=\frac{1}{h_{2}^{2}} C(\underbrace{-2,1,0, \ldots, 0,1}_{N_{y}}) .
$$


Lemma 2.1 ([36]). For a real circulant matrix $A=C\left(a_{0}, a_{1}, \ldots, a_{n-1}\right)$, all eigenvalues of $A$ are given by

$$
f\left(\varepsilon_{k}\right), \quad k=0,1, \ldots, n-1,
$$

where $f(x)=a_{0}+a_{1} x+a_{2} x^{2}+\cdots+a_{n-1} x^{n-1}$, and $\varepsilon_{k}=e^{i \frac{2 k \pi}{n}}$.

Lemma 2.2. For matrices $A_{1}, A_{2}, D_{2}^{x}$ and $D_{2}^{y}$, there exist relations

$$
\begin{array}{ll}
A_{1}=F_{N_{x}}^{H} \Lambda_{1} F_{N_{x}}, & A_{2}=F_{N_{y}}^{H} \Lambda_{2} F_{N_{y}}, \\
D_{2}^{x}=F_{N_{x}}^{H} \Lambda_{3} F_{N_{x}}, & D_{2}^{y}=F_{N_{y}}^{H} \Lambda_{4} F_{N_{y}},
\end{array}
$$

where $F_{N}$ is the discrete Fourier transform with elements $\left(F_{N}\right)_{j, k}=\frac{1}{\sqrt{N}} e^{-i \frac{2 \pi}{N} j k}, F_{N}^{H}$ is the conjugate transpose matrix of $F_{N}$, and

$$
\begin{aligned}
& \Lambda_{1}=\operatorname{diag}\left(\lambda_{A_{1}, 0}, \lambda_{A_{1}, 1}, \ldots, \lambda_{A_{1}, N_{x}-1}\right), \lambda_{A_{1}, j}=-\frac{4}{h_{1}^{2}} \sin ^{2} \frac{j \pi}{N_{x}}, \\
& \Lambda_{2}=\operatorname{diag}\left(\lambda_{A_{2}, 0}, \lambda_{A_{2}, 1}, \ldots, \lambda_{A_{2}, N_{y}-1}\right), \lambda_{A_{2}, k}=-\frac{4}{h_{2}^{2}} \sin ^{2} \frac{k \pi}{N_{y}}, \\
& \Lambda_{3}=\operatorname{diag}\left(\lambda_{D_{2}^{x}, 0}, \lambda_{D_{2}^{x}, 1}, \ldots, \lambda_{D_{2}^{x}, N_{x}-1}\right), \lambda_{D_{2}^{x}, j}= \begin{cases}-\left(j \mu_{1}\right)^{2}, & 0 \leq j \leq N_{x} / 2, \\
-\left(\left(j-N_{x}\right) \mu_{1}\right)^{2}, & N_{x} / 2<j<N_{x},\end{cases} \\
& \Lambda_{4}=\operatorname{diag}\left(\lambda_{D_{2}^{y}, 0}, \lambda_{D_{2}^{y}, 1}, \ldots, \lambda_{D_{2}^{y}, N_{y}-1}\right), \lambda_{D_{2}^{y}, k}= \begin{cases}-\left(k \mu_{2}\right)^{2}, & 0 \leq k \leq N_{y} / 2, \\
-\left(\left(k-N_{y}\right) \mu_{2}\right)^{2}, & N_{y} / 2<k<N_{y} .\end{cases}
\end{aligned}
$$

Furthermore, we have

$$
\begin{aligned}
& 0 \leq-\frac{4}{\pi^{2}} \lambda_{D_{2}^{x}, j} \leq-\lambda_{A_{1}, j} \leq-\lambda_{D_{2}^{x}, j}, \quad \forall 0 \leq j<N_{x}, \\
& 0 \leq-\frac{4}{\pi^{2}} \lambda_{D_{2}^{y}, k} \leq-\lambda_{A_{2}, k} \leq-\lambda_{D_{2}^{y}, k}, \quad \forall 0 \leq k<N_{y} .
\end{aligned}
$$

Proof. According to Lemma 2.1, we have

$$
\lambda_{A_{1}, j}=\frac{1}{h_{1}^{2}}\left(-2+e^{i \frac{2 j \pi}{N_{x}}}+\left(e^{i \frac{2 j \pi}{N_{x}}}\right)^{N_{x}-1}\right)=\frac{1}{h_{1}^{2}}\left(-2+2 \cos \frac{2 j \pi}{N_{x}}\right)=-\frac{4}{h_{1}^{2}} \sin ^{2} \frac{j \pi}{N_{x}} .
$$

Similarly, we can compute $\lambda_{A_{2}, k}$. Since $A_{1}$ and $A_{2}$ are circulant matrices [36], we have (2.7). For the proof of (2.8), please refer to [34].

Using the inequality

$$
0 \leq \frac{2}{\pi} x \leq \sin x \leq x, \forall x \in\left[0, \frac{\pi}{2}\right]
$$

we readily deduce $(2.9)-(2.10)$.

Lemma 2.3. For $A \in \mathbb{C}_{N_{x} \times N_{x}}, B \in \mathbb{C}_{N_{y} \times N_{y}}$, and $u, v \in \mathbb{V}_{h}$, there exist identities

$$
\begin{aligned}
& (A u, v)_{h}=\left(u, A^{H} v\right)_{h}, \\
& (u B, v)_{h}=\left(u, v B^{H}\right)_{h} .
\end{aligned}
$$

Lemma 2.4. For any grid function $u \in \mathbb{V}_{h}$, the following inequalities hold:

$$
|u|_{h, 1} \leq|u|_{h} \leq \frac{\pi}{2}|u|_{h, 1}
$$


Proof. It follows from Lemmas 2.2 and 2.3 that

$$
\begin{aligned}
\left\|\delta_{x}^{+} u\right\|_{h}^{2} & =\left(-A_{1} u, u\right)_{h}=\left(-F_{N_{x}}^{H} \Lambda_{1} F_{N_{x}} u, u\right)_{h}=\left(-\Lambda_{1} F_{N_{x}} u, F_{N_{x}} u\right)_{h} \\
& =-h_{1} h_{2} \sum_{j=0}^{N_{x}-1} \sum_{k=0}^{N_{y}-1} \lambda_{A_{1}, j}\left|\left(F_{N_{x}} u\right)_{j, k}\right|^{2} \\
& \leq-h_{1} h_{2} \sum_{j=0}^{N_{x}-1} \sum_{k=0}^{N_{y}-1} \lambda_{D_{2}^{x}, j}\left|\left(F_{N_{x}} u\right)_{j, k}\right|^{2}=\left(-D_{2}^{x} u, u\right)_{h} .
\end{aligned}
$$

Similarly, we have

$$
\left\|\delta_{x}^{+} u\right\|_{h}^{2} \geq \frac{4}{\pi^{2}}\left(-D_{2}^{x} u, u\right)_{h}
$$

and

$$
\frac{4}{\pi^{2}}\left(-u D_{2}^{y}, u\right)_{h} \leq\left\|\delta_{y}^{+} u\right\|_{h}^{2} \leq\left(-u D_{2}^{y}, u\right)_{h} .
$$

Eqs. (2.14)-(2.16) imply (2.13).

\section{Solution existence and conservation of the scheme}

In this section, we show scheme (2.4) is solvable and it conserves the total mass and energy at the discrete level. Then, we give an a priori estimate for the scheme. First, we introduce some useful lemmas.

Lemma 3.1. For approximation $U^{n} \in \mathbb{V}_{h}$, there exist identities:

$$
\begin{gathered}
\operatorname{Im}\left(D_{2}^{x} U^{n+1 / 2}+U^{n+1 / 2} D_{2}^{y}, U^{n+1 / 2}\right)_{h}=0, \\
\operatorname{Re}\left(D_{2}^{x} U^{n+1 / 2}+U^{n+1 / 2} D_{2}^{y}, \delta_{t}^{+} U^{n}\right)_{h}=-\frac{1}{2 \tau}\left(\left|U^{n+1}\right|_{h}^{2}-\left|U^{n}\right|_{h}^{2}\right),
\end{gathered}
$$

where $\operatorname{Im}(s)$ " and "Re(s)" are the imaginary and the real part of a complex number s, respectively.

Proof. From definition (2.6), we have

$$
\operatorname{Im}\left(D_{2}^{x} U^{n+1 / 2}+U^{n+1 / 2} D_{2}^{y}, U^{n+1 / 2}\right)_{h}=\operatorname{Im}\left(-\left|U^{n+1 / 2}\right|_{h}^{2}\right)=0 .
$$

Using Lemma 2.3 and symmetry of real matrix $D_{2}^{x}$, we obtain

$$
\begin{aligned}
\operatorname{Re}\left(D_{2}^{x} U^{n+1 / 2}, \delta_{t}^{+} U^{n}\right)_{h} & =\frac{1}{2 \tau} \operatorname{Re}\left(D_{2}^{x} U^{n+1}+D_{2}^{x} U^{n}, U^{n+1}-U^{n}\right)_{h} \\
& =\frac{1}{2 \tau}\left(\left(D_{2}^{x} U^{n+1}, U^{n+1}\right)_{h}-\left(D_{2}^{x} U^{n}, U^{n}\right)_{h}\right) .
\end{aligned}
$$

Similarly, we have

$$
\operatorname{Re}\left(U^{n+1 / 2} D_{2}^{y}, \delta_{t}^{+} U^{n}\right)_{h}=\frac{1}{2 \tau}\left(\left(U^{n+1} D_{2}^{y}, U^{n+1}\right)_{h}-\left(U^{n} D_{2}^{y}, U^{n}\right)_{h}\right) .
$$

Adding (3.3) and (3.4) gives (3.2).

Lemma $3.2([56])$. For any grid function $u \in \mathbb{V}_{h}$, the following inequality holds

$$
\|u\|_{h, 4}^{4} \leq\|u\|_{h}^{2}\left(2|u|_{h, 1}+\frac{1}{l}\|u\|_{h}\right)^{2},
$$

where $l=\min \left\{l_{1}, l_{2}\right\}$. 


\subsection{Existence}

Lemma 3.3 (Browder Fixed Point Theorem $[2,11])$. Let $(H,\langle\cdot, \cdot\rangle)$ be a finite dimensional inner product space, $\|\cdot\|$ the associated norm, and $g: H \rightarrow H$ a continuous function. If

$$
\exists \alpha>0, \forall z \in H,\|z\|=\alpha, \text { s. t. } \operatorname{Re}\langle g(z), z\rangle \geq 0,
$$

there exists a $z^{*} \in H,\left\|z^{*}\right\| \leq \alpha$ such that $g\left(z^{*}\right)=0$.

Theorem 3.4. The nonlinear equation system in scheme (2.4) is solvable.

Proof. For a fixed $n,(2.5)$ can be written as

$$
\begin{gathered}
U^{n+1 / 2}-U^{n}-i \frac{\tau}{2}\left[D_{2}^{x} U^{n+1 / 2}+U^{n+1 / 2} D_{2}^{y}+G\left(U^{n}, U^{n+1 / 2}\right)\right]=0 \\
U^{n} \in \mathbb{V}_{h}, \quad U^{n+1 / 2} \in \mathbb{V}_{h},
\end{gathered}
$$

where $G\left(U^{n}, U^{n+1 / 2}\right)=\left(G\left(U_{j, k}^{n}, U_{j, k}^{n+1 / 2}\right)\right)=\left(\frac{\beta}{2}\left(\left|U_{j, k}^{n}\right|^{2}+\left|2 U_{j, k}^{n+1 / 2}-U_{j, k}^{n}\right|^{2}\right) U_{j, k}^{n+1 / 2}\right)$. We define a mapping $\mathcal{F}: \mathbb{V}_{h} \rightarrow \mathbb{V}_{h}$ as follows

$$
\mathcal{F} w=w-U^{n}-i \frac{\tau}{2}\left[D_{2}^{x} w+w D_{2}^{y}+G\left(U^{n}, w\right)\right],
$$

which is obviously continuous. By a straightforward calculation, we obtain

$$
\left(D_{2}^{x} w+w D_{2}^{y}+G\left(U^{n}, w\right), w\right)_{h}=-|w|_{h}^{2}+\frac{\beta}{2}\left(\left|U^{n}\right|^{2}+\left|2 w-U^{n}\right|^{2},|w|^{2}\right)_{h},
$$

which is a real number. Computing the inner product of (3.8) with $w$ and taking the real part, then using (3.9) and Cauchy-Schwarz inequality, we have

$$
\begin{aligned}
\operatorname{Re}(\mathcal{F} w, w)_{h} & =\|w\|_{h}^{2}-\operatorname{Re}\left(U^{n}, w\right)_{h} \\
& \geq\|w\|_{h}^{2}-\left|\left(U^{n}, w\right)_{h}\right| \\
& \geq\|w\|_{h}^{2}-\left\|U^{n}\right\|_{h}\|w\|_{h} \\
& \geq \frac{1}{2}\left(\|w\|_{h}^{2}-\left\|U^{n}\right\|_{h}^{2}\right) .
\end{aligned}
$$

Hence, taking $\alpha=\sqrt{\left\|U^{n}\right\|_{h}^{2}+1}$, for $\|w\|_{h}=\alpha$, we have $\operatorname{Re}(\mathcal{F} w, w)_{h} \geq \frac{1}{2}$. Thus, the existence of $U^{n+1 / 2}$ follows from Lemma 3.3 and consequently the existence of $U^{n+1}$ is established.

\subsection{Conservation}

Theorem 3.5. Scheme (2.4) is conservative in the sense that

$$
\begin{aligned}
& Q^{n}=Q^{0}, t_{n} \in \Omega_{\tau}, \\
& E^{n}=E^{0}, t_{n} \in \Omega_{\tau},
\end{aligned}
$$

where

$$
Q^{n}=\left\|U^{n}\right\|_{h}^{2}, \quad E^{n}=\left|U^{n}\right|_{h}^{2}-\frac{\beta}{2}\left\|U^{n}\right\|_{h, 4}^{4},
$$

are the discrete total mass and discrete total energy, respectively. 
Proof. Computing the discrete inner product of (2.5) with $U^{n+1 / 2}$, then taking the imaginary part, we obtain

$$
\frac{1}{2 \tau}\left(\left\|U^{n+1}\right\|_{h}^{2}-\left\|U^{n}\right\|_{h}^{2}\right)=0, t_{n} \in \Omega_{\tau},
$$

where Lemma 3.1 is used. This gives (3.11).

Computing the discrete inner product of $(2.5)$ with $\delta_{t}^{+} U^{n}$, then taking the real part, we obtain

$$
-\frac{1}{2 \tau}\left[\left(\left|U^{n+1}\right|_{h}^{2}-\frac{\beta}{2}\left\|U^{n+1}\right\|_{h, 4}^{4}\right)-\left(\left|U^{n}\right|_{h}^{2}-\frac{\beta}{2}\left\|U^{n}\right\|_{h, 4}^{4}\right)\right]=0, t_{n} \in \Omega_{\tau},
$$

where Lemma 3.1 is used. This yields (3.12).

\subsection{A priori estimate}

Lemma 3.6. If one of the following two conditions are satisfied:

$$
\begin{aligned}
& \text { (a) } \varphi \in H_{p}^{1}(\Omega), \quad \beta<0, \\
& \text { (b) } \varphi \in H_{p}^{1}(\Omega), \quad\|\varphi\|_{h}^{2} \leq \frac{2-\widetilde{\varepsilon}}{4(1+\varepsilon) \beta}, \quad \beta>0,
\end{aligned}
$$

where $\varphi$ is the initial condition in (1.3), $\varepsilon$ and $\widetilde{\varepsilon}$ are two positive numbers which can be arbitrarily small, there exists a constant $C$ so that for the solution calculated using scheme (2.4):

$$
\left\|U^{n}\right\|_{h} \leq C, \quad\left|U^{n}\right|_{h, 1} \leq C, \quad t_{n} \in \Omega_{\tau} .
$$

Proof. It follows from (3.11) that

$$
\left\|U^{n}\right\|_{h}=\|\varphi\|_{h} \leq C, \quad t_{n} \in \Omega_{\tau} .
$$

First, under condition $(a)$, we obtain directly from (3.12) that

$$
\left|U^{n}\right|_{h} \leq C, \quad t_{n} \in \Omega_{\tau}
$$

This, together with Lemma 2.4, gives

$$
\left|U^{n}\right|_{h, 1} \leq C, \quad t_{n} \in \Omega_{\tau} .
$$

Second, under condition (b), using Lemma 3.2 and Theorem 3.5, we obtain

$$
\begin{aligned}
\left\|U^{n}\right\|_{h, 4}^{4} & \leq\left\|U^{n}\right\|_{h}^{2}\left(2\left|U^{n}\right|_{h, 1}+\frac{1}{l}\left\|U^{n}\right\|_{h}\right)^{2} \leq 4(1+\varepsilon)\left\|U^{n}\right\|_{h}^{2}\left|U^{n}\right|_{h, 1}^{2}+\left(1+\varepsilon^{-1}\right) \frac{1}{l^{2}}\left\|U^{n}\right\|_{h}^{4} \\
& =4(1+\varepsilon)\|\varphi\|_{h}^{2}\left|U^{n}\right|_{h, 1}^{2}+\left(1+\varepsilon^{-1}\right) \frac{1}{l^{2}}\|\varphi\|_{h}^{4} \leq \frac{2-\widetilde{\varepsilon}}{\beta}\left|U^{n}\right|_{h, 1}^{2}+\left(1+\varepsilon^{-1}\right) \frac{1}{l^{2}}\|\varphi\|_{h}^{4},
\end{aligned}
$$

where the inequality $(a+b)^{2} \leq(1+\varepsilon) a^{2}+\left(1+\varepsilon^{-1}\right) b^{2}$ is used. This, together with Lemma 2.4 and Theorem 3.5, gives

$$
\begin{aligned}
\left|U^{n}\right|_{h, 1}^{2} & \leq\left|U^{n}\right|_{h}^{2}=E^{0}+\frac{\beta}{2}\left\|U^{n}\right\|_{h, 4}^{4} \\
& \leq \frac{2-\widetilde{\varepsilon}}{2}\left|U^{n}\right|_{h, 1}^{2}+C,
\end{aligned}
$$

which implies $\left|U^{n}\right|_{h, 1} \leq C, \quad t_{n} \in \Omega_{\tau}$. 


\section{Convergence of the scheme}

Different from the finite difference method, error estimates of spectral or pseudo-spectral method often rely on the projection error and interpolation error in the spectral space. In this section, we present some basic approximation results for the trigonometric polynomials and prove the unconditional convergence of scheme $(2.4)$ in the discrete $L^{2}$ norm.

For simplicity, we let $\Omega=[0,2 \pi] \times[0,2 \pi], L^{2}(\Omega)$ with the inner product $(\cdot, \cdot)$ and the norm $\|\cdot\|$ defined previously. For any positive integer $r$, the semi-norm and the norm of $H^{r}(\Omega)$ are denoted by $|\cdot|_{r}$ and $\|\cdot\|_{r}$, respectively. Let $C_{p}^{\infty}(\Omega)$ be the set of infinitely differentiable functions with $(2 \pi, 2 \pi)$-period. $H_{p}^{r}(\Omega)$ is the closure of $C_{p}^{\infty}(\Omega)$ in $H^{r}(\Omega)$. Let $N_{x}=N_{y}=N$, the interpolation space $S_{N}^{\prime \prime}$ can be written as

$$
S_{N}^{\prime \prime}=\left\{u\left|u(x, y)=\sum_{|l| \leq N / 2} \sum_{|m| \leq N / 2} \frac{\widehat{u}_{l, m}}{c_{l} c_{m}} e_{l, m}, \widehat{u}_{\frac{N}{2}, m}=\widehat{u}_{-\frac{N}{2}, m}, \widehat{u}_{l, \frac{N}{2}}=\widehat{u}_{l,-\frac{N}{2}},\right| l\left|\leq \frac{N}{2},\right| m \mid \leq \frac{N}{2}\right\},
$$

where $e_{l, m}=e^{i(l x+m y)}, c_{l}=\left\{\begin{array}{l}1,|l|<N / 2 \\ 2,|l|=N / 2\end{array}\right.$

We denote

$$
S_{N}=\left\{u \mid u(x, y)=\sum_{|l| \leq N / 2} \sum_{|m| \leq N / 2} \widehat{u}_{l, m} e_{l, m}\right\} .
$$

It is obviously that $S_{N}^{\prime \prime} \subseteq S_{N}$. We denote the orthogonal projection operator as $P_{N}: L^{2}(\Omega) \rightarrow$ $S_{N}$ and recall the interpolation operator $I_{N}: L^{2}(\Omega) \rightarrow S_{N}^{\prime \prime}$. Note that $P_{N}$ and $I_{N}$ satisfy the following properties:

- $P_{N} \partial_{z} u=\partial_{z} P_{N} u, I_{N} \partial_{z} u \neq \partial_{z} I_{N} u, z=x$ or $y$;

- $P_{N} u=u, \forall u \in S_{N} ; I_{N} u=u, \forall u \in S_{N}^{\prime \prime}$.

Lemma 4.1. For any $u \in S_{N}^{\prime \prime},\|u\| \leq\|u\|_{h} \leq 2\|u\|$.

Proof. For any $u \in S_{N}^{\prime \prime}$, noticing that $\left(e_{l, m}, e_{j, k}\right)=4 \pi^{2} \delta_{l}^{j} \delta_{m}^{k}$, we obtain

$$
\begin{aligned}
\|u\|^{2} & =\left(\sum_{|l| \leq N / 2} \sum_{|m| \leq N / 2} \frac{\widehat{u}_{l, m}}{c_{l} c_{m}} e_{l, m}, \sum_{|l| \leq N / 2} \sum_{|m| \leq N / 2} \frac{\widehat{u}_{l, m}}{c_{l} c_{m}} e_{l, m}\right) \\
& =4 \pi^{2} \sum_{|l| \leq N / 2} \sum_{|m| \leq N / 2} \frac{\left|\widehat{u}_{l, m}\right|^{2}}{\left(c_{l} c_{m}\right)^{2}} \\
& =4 \pi^{2}\left(\frac{1}{4}\left|\widehat{u}_{N / 2, N / 2}\right|^{2}+\frac{1}{2} \sum_{|l|<N / 2}\left|\widehat{u}_{l, N / 2}\right|^{2}+\frac{1}{2} \sum_{|m|<N / 2}\left|\widehat{u}_{N / 2, m}\right|^{2}+\sum_{|l|<N / 2} \sum_{|m|<N / 2}\left|\widehat{u}_{l, m}\right|^{2}\right) .
\end{aligned}
$$

Similarly, noticing that

$$
\left(e_{l, m}, e_{j, k}\right)_{N}= \begin{cases}4 \pi^{2}, & l \equiv j(\bmod N) \text { and } m \equiv k(\bmod N), \\ 0, & \text { other }\end{cases}
$$

we obtain

$$
\|u\|_{h}^{2}=4 \pi^{2}\left(\left|\widehat{u}_{N / 2, N / 2}\right|^{2}+\sum_{|l|<N / 2}\left|\widehat{u}_{l, N / 2}\right|^{2}+\sum_{|m|<N / 2}\left|\widehat{u}_{N / 2, m}\right|^{2}+\sum_{|l|<N / 2} \sum_{|m|<N / 2}\left|\widehat{u}_{l, m}\right|^{2}\right) .
$$


Combining (4.1) and (4.2), we have

$$
\|u\| \leq\|u\|_{h} \leq 2\|u\|
$$

Lemma $4.2([16])$. If $0 \leq l \leq r$ and $u \in H_{p}^{r}(\Omega)$,

$$
\begin{aligned}
& \left\|P_{N} u-u\right\|_{l} \leq C N^{l-r}|u|_{r}, \\
& \left\|P_{N} u\right\|_{l} \leq C\|u\|_{l} ;
\end{aligned}
$$

if, in addition, $r>1$,

$$
\begin{aligned}
& \left\|I_{N} u-u\right\|_{l} \leq C N^{l-r}|u|_{r} \\
& \left\|I_{N} u\right\|_{l} \leq C\|u\|_{l} .
\end{aligned}
$$

Lemma 4.3. For $u \in H_{p}^{r}(\Omega), r>1$, let $u^{*}=P_{N-2} u$, then $\left\|u^{*}-u\right\|_{h} \leq C N^{-r}|u|_{r}$.

Proof. From Lemma 4.1 and Lemma 4.2, we obtain

$$
\begin{aligned}
\left\|u^{*}-u\right\|_{h} & =\left\|I_{N}\left(u^{*}-u\right)\right\|_{h} \\
& \leq 2\left\|I_{N}\left(u^{*}-u\right)\right\| \\
& \leq C\left\|u^{*}-u\right\| \\
& \leq C(N-2)^{-r}|u|_{r}=C\left(1-\frac{2}{N}\right)^{-r} N^{-r}|u|_{r} \\
& \leq C N^{-r}|u|_{r},
\end{aligned}
$$

where the last inequality holds if $N \geq 4$.

Lemma 4.4 ([52]). For any complex numbers $U, V, u, v$, the following inequality holds

$$
\left.|| U\right|^{2} V-|u|^{2} v \mid \leq(\max \{|U|,|V|,|u|,|v|\})^{2} \cdot(2|U-u|+|V-v|) .
$$

Lemma 4.5 (Gronwall Inequality [61]). Suppose that the nonnegative discrete function $\left\{\omega^{n} \mid n=\right.$ $\left.0,1,2, \ldots, N_{t} ; N_{t} \tau=T\right\}$ satisfies the inequality

$$
\omega^{n}-\omega^{n-1} \leq A \tau \omega^{n}+B \tau \omega^{n-1}+C_{n} \tau,
$$

where $A, B$ and $C_{n}\left(n=1,2, \ldots, N_{t}\right)$ are nonnegative constants. Then

$$
\max _{1 \leq n \leq N_{t}} \omega^{n} \leq\left(\omega^{0}+\tau \sum_{l=1}^{N_{t}} C_{l}\right) e^{2(A+B) T},
$$

where $\tau$ is sufficiently small, such that $(A+B) \tau \leq \frac{N_{t}-1}{2 N_{t}}\left(N_{t}>1\right)$.

Theorem 4.6. Under assumptions $(a)$ and $(b)$ of Lemma 3.6, if

$$
u(x, y, t) \in C^{3}\left(0, T ; H_{p}^{r}(\Omega)\right), r>1,
$$

the Fourier pseudo-spectral solution of scheme (2.4) converges, without any restrictions on the grid ratio, to the solution of the initial-periodic boundary value problem (1.1)-(1.3) in the order of $O\left(N^{-r}+\tau^{2}\right)$ in the discrete $L^{2}$ norm. 
Proof. We denote

$$
u^{*}=P_{N-2} u, \quad f=f(u)=\beta|u|^{2} u, \quad f^{*}=P_{N-2} f .
$$

The projection of Eq. (1.1) is written as

$$
i \partial_{t} u^{*}+\partial_{x x} u^{*}+\partial_{y y} u^{*}+f^{*}=0
$$

We define

$$
\eta_{j, k}^{n}=i \delta_{t}^{+} u_{j, k}^{* n}+\left(D_{2}^{x} u^{* n+1 / 2}\right)_{j, k}+\left(u^{* n+1 / 2} D_{2}^{y}\right)_{j, k}+f_{j, k}^{* n+1 / 2} .
$$

Noticing $u^{*} \in S_{J}^{\prime \prime}, \partial_{x x} u^{*}\left(x_{j}, y_{k}, t_{n}\right)=\left(D_{2}^{x} u^{* n}\right)_{j, k}, \partial_{y y} u^{*}\left(x_{j}, y_{k}, t_{n}\right)=\left(u^{* n} D_{2}^{y}\right)_{j, k}$, we obtain

$$
\eta_{j, k}^{n}=i\left(\delta_{t}^{+} u_{j, k}^{* n}-\partial_{t} u_{j, k}^{* n+1 / 2}\right) .
$$

Using the Taylor expansion, we obtain

$$
\left|\eta_{j, k}^{n}\right| \leq C \tau^{2}
$$

for some constant $C$.

Denote $e_{j, k}^{n}=u_{j, k}^{* n}-U_{j, k}^{n}$. Subtracting (2.4) from (4.5) yields the following error equation

$$
\begin{aligned}
& i \delta_{t}^{+} e^{n}+D_{2}^{x} e^{n+1 / 2}+e^{n+1 / 2} D_{2}^{y}+G^{n}=\eta^{n}, \\
& e^{0}=u^{* 0}-u^{0},
\end{aligned}
$$

where

$$
G_{j, k}^{n}=f_{j, k}^{* n+1 / 2}-F\left(U_{j, k}^{n}, U_{j, k}^{n+1}\right) .
$$

Denoting

$$
\begin{aligned}
& \left(G_{1}\right)_{j, k}^{n}=f_{j, k}^{* n+1 / 2}-f_{j, k}^{n+1 / 2}, \quad\left(G_{2}\right)_{j, k}^{n}=f_{j, k}^{n+1 / 2}-F\left(u_{j, k}^{n}, u_{j, k}^{n+1}\right), \\
& \left(G_{3}\right)_{j, k}^{n}=F\left(u_{j, k}^{n}, u_{j, k}^{n+1}\right)-F\left(u_{j, k}^{* n}, u_{j, k}^{* n+1}\right), \quad\left(G_{4}\right)_{j, k}^{n}=F\left(u_{j, k}^{* n}, u_{j, k}^{* n+1}\right)-F\left(U_{j, k}^{n}, U_{j, k}^{n+1}\right),
\end{aligned}
$$

we have

$$
G_{j, k}^{n}=\left(G_{1}\right)_{j, k}^{n}+\left(G_{2}\right)_{j, k}^{n}+\left(G_{3}\right)_{j, k}^{n}+\left(G_{4}\right)_{j, k}^{n} .
$$

According to Lemma 4.3, we have

$$
\left\|G_{1}^{n}\right\|_{h} \leq C N^{-r}
$$

Using the Taylor expansion with an integral remainder, there exist complex numbers $c_{k},\left|c_{k}\right| \leq$ $C, k=1,2,3$ such that

$$
\begin{aligned}
& \frac{1}{2}\left(\left|u^{n}\right|^{2} u^{n}+\left|u^{n+1}\right|^{2} u^{n+1}\right)=\left|u\left(x, y, t_{n+1 / 2}\right)\right|^{2} u\left(x, y, t_{n+1 / 2}\right)+c_{1} \tau^{2}, \\
& \frac{1}{2}\left(\left|u^{n}\right|^{2}+\left|u^{n+1}\right|^{2}\right)=\left|u\left(x, y, t_{n+1 / 2}\right)\right|^{2}+c_{2} \tau^{2}, \\
& u^{n+1 / 2}=u\left(x, y, t_{n+1 / 2}\right)+c_{3} \tau^{2} .
\end{aligned}
$$

Therefore we have

$$
\left|\left(G_{2}\right)_{j, k}^{n}\right|=\left|\frac{\beta}{2}\left(\left|u_{j, k}^{n}\right|^{2} u_{j, k}^{n}+\left|u_{j, k}^{n+1}\right|^{2} u_{j, k}^{n+1}\right)-\frac{\beta}{2}\left(\left|u_{j, k}^{n}\right|^{2}+\left|u_{j, k}^{n+1}\right|^{2}\right) u_{j, k}^{n+1 / 2}\right| \leq C \tau^{2} .
$$

From Lemma 4.4, we deduce

$$
\left|\left(G_{3}\right)_{j, k}^{n}\right| \leq C\left(\left|u_{j, k}^{n}-u_{j, k}^{* n}\right|+\left|u_{j, k}^{n+1}-u_{j, k}^{* n+1}\right|\right) .
$$


This, together with Lemma 4.3, gives

$$
\left\|G_{3}^{n}\right\|_{h} \leq C N^{-r}
$$

Notice that

$$
\begin{aligned}
\left(G_{4}\right)_{j, k}^{n} & =\frac{\beta}{2}\left(\left|u_{j, k}^{* n}\right|^{2}+\left|u_{j, k}^{* n+1}\right|^{2}\right) u_{j, k}^{* n+1 / 2}-\frac{\beta}{2}\left(\left|U_{j, k}^{n}\right|^{2}+\left|U_{j, k}^{n+1}\right|^{2}\right) U_{j, k}^{n+1 / 2} \\
& =\frac{\beta}{2}\left(\left|u_{j, k}^{* n}\right|^{2}+\left|u_{j, k}^{* n+1}\right|^{2}-\left|U_{j, k}^{n}\right|^{2}-\left|U_{j, k}^{n+1}\right|^{2}\right) u_{j, k}^{* n+1 / 2}+\frac{\beta}{2}\left(\left|U_{j, k}^{n}\right|^{2}+\left|U_{j, k}^{n+1}\right|^{2}\right) e_{j, k}^{n+1 / 2} \\
& :=\phi_{j, k}^{n}+\psi_{j, k}^{n},
\end{aligned}
$$

where

$$
\begin{gathered}
\phi_{j, k}^{n}=\frac{\beta}{2}\left(\left|u_{j, k}^{* n}\right|^{2}+\left|u_{j, k}^{* n+1}\right|^{2}-\left|u_{j, k}^{* n}-e_{j, k}^{n}\right|^{2}-\left|u_{j, k}^{* n+1}-e_{j, k}^{n+1}\right|^{2}\right) u_{j, k}^{* n+1 / 2} \\
=\frac{\beta}{2}\left(u_{j, k}^{* n} \bar{e}_{j, k}^{n}+{\overline{u^{*}}}_{j, k}^{n} e_{j, k}^{n}+u_{j, k}^{* n+1} \bar{e}_{j, k}^{n+1}+{\overline{u^{*}}}_{j, k}^{n+1} e_{j, k}^{n+1}-\left|e_{j, k}^{n}\right|^{2}-\left|e_{j, k}^{n+1}\right|^{2}\right) u_{j, k}^{* n+1 / 2}, \\
\psi_{j, k}^{n}=\frac{\beta}{2}\left(\left|U_{j, k}^{n}\right|^{2}+\left|U_{j, k}^{n+1}\right|^{2}\right) e_{j, k}^{n+1 / 2} .
\end{gathered}
$$

Computing the discrete inner product of (4.8) with $e^{n+1 / 2}$, then taking the imaginary part, we obtain

$$
\frac{1}{2 \tau}\left(\left\|e^{n+1}\right\|_{h}^{2}-\left\|e^{n}\right\|_{h}^{2}\right)+\operatorname{Im}\left(G_{1}^{n}+G_{2}^{n}+G_{3}^{n}+G_{4}^{n}, e^{n+1 / 2}\right)_{h}=\operatorname{Im}\left(\eta^{n}, e^{n+1 / 2}\right)_{h},
$$

where Lemma 3.1 was used. Using Cauchy-Schwartz inequality, we obtain

$$
\begin{aligned}
& \left|\left(G_{s}^{n}, e^{n+1 / 2}\right)_{h}\right| \leq \frac{1}{2}\left\|G_{s}^{n}\right\|_{h}^{2}+\frac{1}{4}\left(\left\|e^{n}\right\|_{h}^{2}+\left\|e^{n+1}\right\|_{h}^{2}\right), s=1,2,3, \\
& \left|\operatorname{Im}\left(G_{4}^{n}, e^{n+1 / 2}\right)_{h}\right|=\left|\operatorname{Im}\left(\phi^{n}, e^{n+1 / 2}\right)_{h}\right| \leq C\left(\left\|e^{n}\right\|_{h}^{2}+\left\|e^{n+1}\right\|_{h}^{2}+\left\|e^{n}\right\|_{h, 4}^{4}+\left\|e^{n+1}\right\|_{h, 4}^{4}\right), \\
& \left|\left(\eta^{n}, e^{n+1 / 2}\right)_{h}\right| \leq \frac{1}{2}\left\|\eta^{n}\right\|_{h}^{2}+\frac{1}{4}\left(\left\|e^{n}\right\|_{h}^{2}+\left\|e^{n+1}\right\|_{h}^{2}\right) .
\end{aligned}
$$

It follows from Lemma 3.6, Lemma 4.1 and Lemma 4.2 that

$$
\left\|e^{n}\right\|_{h}=\left\|u^{* n}-U^{n}\right\|_{h} \leq\left\|u^{* n}\right\|_{h}+\left\|U^{n}\right\|_{h} \leq 2\left\|u^{* n}\right\|+\left\|U^{n}\right\|_{h} \leq C .
$$

Similarly, we obtain

$$
\left|e^{n}\right|_{h, 1} \leq C
$$

Eqs. (4.24)-(4.25), together with Lemma 3.2, give

$$
\left\|e^{n}\right\|_{h, 4}^{4} \leq C\left\|e^{n}\right\|_{h}^{2}
$$

This, together with (4.22), gives

$$
\left|\operatorname{Im}\left(G_{4}^{n}, e^{n+1 / 2}\right)_{h}\right| \leq C\left(\left\|e^{n}\right\|_{h}^{2}+\left\|e^{n+1}\right\|_{h}^{2}\right) .
$$

Substituting (4.21), (4.23) and (4.27) into (4.20) yields

$$
\frac{1}{2 \tau}\left(\left\|e^{n+1}\right\|_{h}^{2}-\left\|e^{n}\right\|_{h}^{2}\right) \leq C\left(\left\|e^{n}\right\|_{h}^{2}+\left\|e^{n+1}\right\|_{h}^{2}\right)+\frac{1}{2}\left(\left\|G_{1}^{n}\right\|_{h}^{2}+\left\|G_{2}^{n}\right\|_{h}^{2}+\left\|G_{3}^{n}\right\|_{h}^{2}+\left\|\eta^{n}\right\|_{h}^{2}\right) .
$$


This, together with Lemma 4.5 and (4.10), (4.14), (4.16), gives that, for a sufficiently small $\tau$,

$$
\left\|e^{n}\right\|_{h}^{2} \leq\left(\left\|e^{0}\right\|_{h}^{2}+C T\left(N^{-2 r}+\tau^{4}\right)\right) e^{4 C T}
$$

This, together with

$$
\left\|e^{0}\right\|_{h}=\left\|u^{* 0}-u^{0}\right\|_{h} \leq C N^{-r}
$$

gives

$$
\left\|e^{n}\right\|_{h} \leq C\left(N^{-r}+\tau^{2}\right)
$$

According to Lemma 4.3 and (4.30), we obtain

$$
\left\|u^{n}-U^{n}\right\|_{h} \leq\left\|u^{n}-u^{* n}\right\|_{h}+\left\|u^{* n}-U^{n}\right\|_{h} \leq C\left(N^{-r}+\tau^{2}\right)
$$

This completes the proof.

\section{$5 \quad$ Numerical examples}

In this section, we present some numerical examples to substantiate our theoretical analyses on convergence and conservation of mass and energy.

First, we present a fast approach to solving nonlinear system (2.5) [49]. At time step $n$, the nonlinear system defined in scheme $(2.5)$ is computed by the following fixed-point iteration method

$$
i \frac{U_{s+1}^{n+1}-U^{n}}{\tau}+D_{2}^{x} \frac{U_{s+1}^{n+1}+U^{n}}{2}+\frac{U_{s+1}^{n+1}+U^{n}}{2} D_{2}^{y}+F\left(U^{n}, U_{s}^{n+1}\right)=0,
$$

where the initial iteration $U_{0}^{n+1}=U^{n}$. We iterate the solution until the following criteria is satisfied

$$
\left\|U_{s+1}^{n+1}-U_{s}^{n+1}\right\|_{h, \infty}<10^{-14} .
$$

For iteration step $s$, system (5.1) can be simplified into

$$
i U_{s+1}^{n+1}+\frac{\tau}{2} D_{2}^{x} U_{s+1}^{n+1}+\frac{\tau}{2} U_{s+1}^{n+1} D_{2}^{y}=B
$$

where $B=i U^{n}-\frac{\tau}{2} D_{2}^{x} U^{n}-\frac{\tau}{2} U^{n} D_{2}^{y}-\tau F\left(U^{n}, U_{s}^{n+1}\right)$. Let $\widetilde{U}_{s+1}^{n+1}=F_{N_{x}} U_{s+1}^{n+1} F_{N_{y}}^{H}, \widetilde{B}=F_{N_{x}} B F_{N_{y}}^{H}$. According to Lemma 2.2 and $F_{N}^{H} F_{N}=I$, multiplying $F_{N_{x}}$ and $F_{N_{y}}^{H}$ on both sides of (5.2), respectively, we have

$$
i \widetilde{U}_{s+1}^{n+1}+\frac{\tau}{2} \Lambda_{3} \widetilde{U}_{s+1}^{n+1}+\frac{\tau}{2} \widetilde{U}_{s+1}^{n+1} \Lambda_{4}=\widetilde{B}
$$

which gives

$$
\left(\widetilde{U}_{s+1}^{n+1}\right)_{j, k}=\frac{\widetilde{B}_{j, k}}{i+\frac{\tau}{2} \lambda_{D_{2}^{x}, j}+\frac{\tau}{2} \lambda_{D_{2}^{y}, k}} .
$$

Solving the above equations gives the matrix $\widetilde{U}_{s+1}^{n+1}$. Using the relation $U_{s+1}^{n+1}=F_{N_{x}}^{H} \widetilde{U}_{s+1}^{n+1} F_{N_{y}}$ yields the solution matrix $U_{s+1}^{n+1}$. Note that we can apply the Fast Fourier Transform (FFT) algorithm to the above process.

Example 5.1. We consider the 2D NLS equation (1.1) with a progressive plane wave solution $[58]$

$$
u(x, y, t)=A \exp \left(i\left(k_{1} x+k_{2} y-\omega t\right)\right)
$$

where $\omega=k_{1}^{2}+k_{2}^{2}-\beta|A|^{2}$. 
Firstly, for the convergence test, the problem is solved on domain $[0,2 \pi] \times[0,2 \pi]$ until $T=1$ with $A=1, k_{1}=k_{2}=1, \beta=-1$. The convergence order is calculated using the formula

$$
\text { Order }=\frac{\ln \left(\text { error }_{1} / \text { error }_{2}\right)}{\ln \left(\delta_{1} / \delta_{2}\right)},
$$

where $\delta_{l}$, error,$(l=1,2)$ are step size and the error with step size $\delta_{l}$, respectively. Table 1 shows that the spatial error is very small and almost negligible, and the error is dominated by the time discretization error. It confirms that, for the sufficiently smooth problem, the Fourier pseudo-spectral method is of arbitrary order of accuracy. Table 2 clearly indicates that the method is of the second-order in time. These numerical results confirm the result in Theorem 4.6. The maximum iterations and the total CPU time are also given in Tables 1-2, which show that our algorithm is very efficient in this practical computation. It is worth noting that the maximum number of iterations reduces as time step $\tau$ decreases.

Table 1: Convergence test in space with $\tau=1.0 e-6$ at $T=1$.

\begin{tabular}{lllll}
\hline$N_{x} \times N_{y}$ & Error & & Maximum iterations & CPU(s) \\
\cline { 2 - 3 } & $L^{\infty}$ & $L^{2}$ & & \\
\hline $8 \times 8$ & $1.2341 \mathrm{e}-10$ & $6.2680 \mathrm{e}-10$ & 3 & 59.4 \\
$16 \times 16$ & $4.6966 \mathrm{e}-11$ & $2.2571 \mathrm{e}-10$ & 3 & 138.6 \\
$32 \times 32$ & $7.9636 \mathrm{e}-11$ & $4.5296 \mathrm{e}-10$ & 3 & 359.8 \\
$64 \times 64$ & $1.4757 \mathrm{e}-11$ & $5.0357 \mathrm{e}-11$ & 3 & 1386.0 \\
$128 \times 128$ & $3.7748 \mathrm{e}-11$ & $1.8009 \mathrm{e}-10$ & 3 & 3765.4 \\
\hline
\end{tabular}

Table 2: Convergence test in time with $N_{x}=N_{y}=64$ at $T=1$.

\begin{tabular}{|c|c|c|c|c|c|c|}
\hline \multirow[t]{2}{*}{$\tau$} & \multicolumn{2}{|l|}{ Error } & \multicolumn{2}{|l|}{ Order } & \multirow[t]{2}{*}{ Maximum iterations } & \multirow[t]{2}{*}{$\mathrm{CPU}(\mathrm{s})$} \\
\hline & $L^{\infty}$ & $L^{2}$ & $L^{\infty}$ & $L^{2}$ & & \\
\hline$\overline{0.02}$ & $8.9951 \mathrm{e}-04$ & $5.6518 \mathrm{e}-03$ & - & - & 9 & 0.3 \\
\hline 0.01 & $2.2497 \mathrm{e}-04$ & $1.4135 \mathrm{e}-03$ & 1.9994 & 1.9994 & 8 & 0.6 \\
\hline 0.005 & $5.6248 \mathrm{e}-05$ & $3.5342 \mathrm{e}-04$ & 1.9999 & 1.9999 & 7 & 1.0 \\
\hline 0.0025 & $1.4062 \mathrm{e}-05$ & $8.8357 \mathrm{e}-05$ & 2.0000 & 2.0000 & 6 & 1.7 \\
\hline 0.00125 & $3.5156 \mathrm{e}-06$ & $2.2089 \mathrm{e}-05$ & 2.0000 & 2.0000 & 5 & 2.7 \\
\hline
\end{tabular}

Secondly, we compare the Fourier pseudo-spectral (FP) method (2.5) with the compact finite difference (CFD) method proposed in [56]. In this comparison, we focus on the longtime behavior of solutions in capturing high-frequency waves. The computations are conducted on $[0,2 \pi] \times[0,2 \pi]$ until $T=10$. Given the spatial grid size $N_{x}=N_{y}=64$ and the parameter values $A=1, k_{1}=k_{2}=4, \beta=-2$, we compute the discrete $L^{2}$ and $L^{\infty}$ errors between the exact solution and the numerical solution obtained by the two methods, respectively, as shown in Fig 1 a-b. For $t \in[0,10]$, the solution error increases linearly over time and the numerical approximate results obtained using the FP method are much better than that of the CFD method. When the time step reduces by one order of magnitude $\left(\tau=1.0 e^{-5}\right)$, the error using the FP method reduces further while that using the CFD does not show much improvement. The errors in the global mass and energy are also plotted in Fig. $1 \mathrm{c}$-d. Notice that the errors in mass and energy 
are very small, despite of a linear growth in the solution, which might be due to the temporal finite differencing. Similar phenomena are shown in Fig 2, where a high-frequency wave with $A=1, k_{1}=k_{2}=8, \beta=-2$ is approximated.

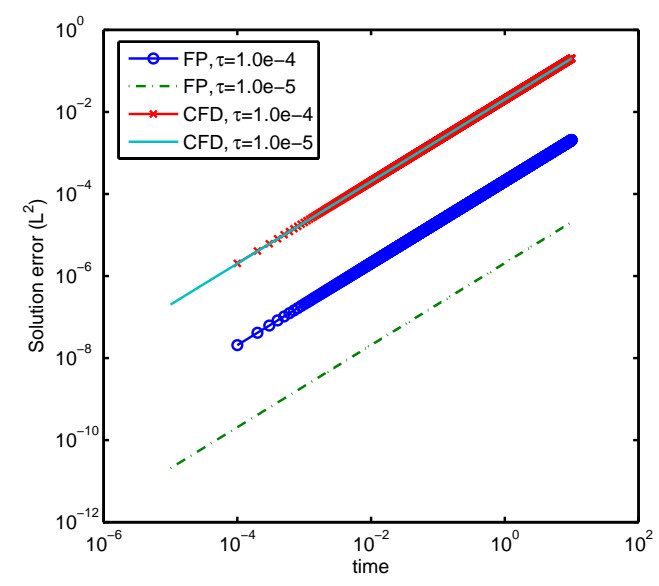

(a) The solution error in $L^{2}$ norm

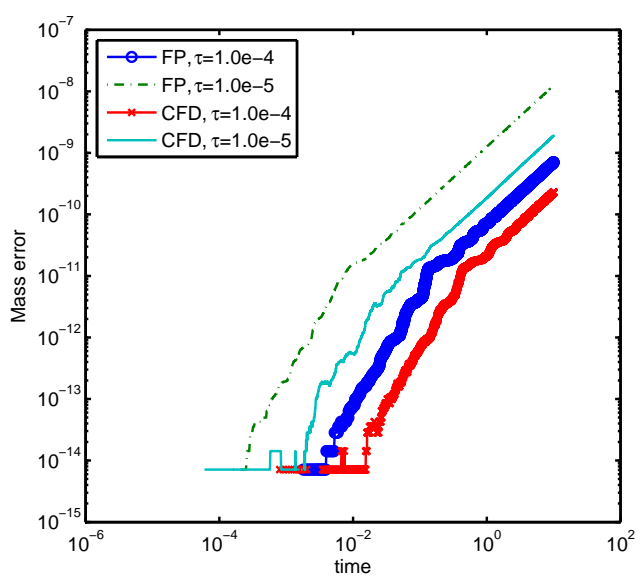

(c) Mass error

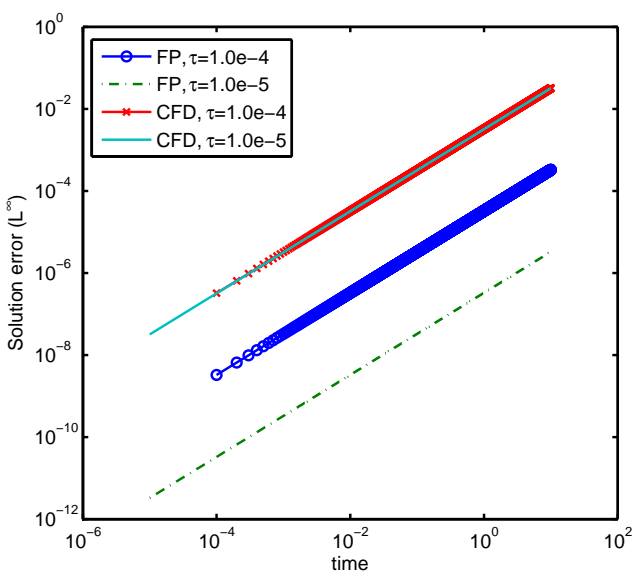

(b) The solution error in $L^{\infty}$ norm

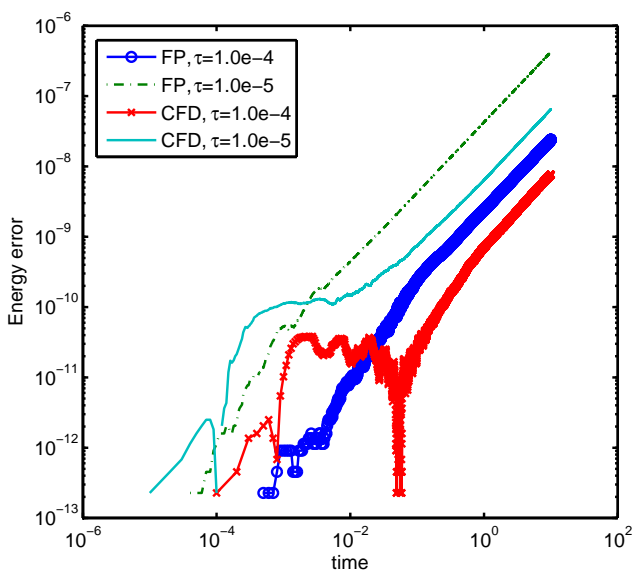

(d) Energy error

Figure 1: Error comparison between the FP and the CFD method $\left(\beta=-2, A=1, k_{1}=k_{2}=4, N_{x}=\right.$ $\left.N_{y}=64\right)$. 


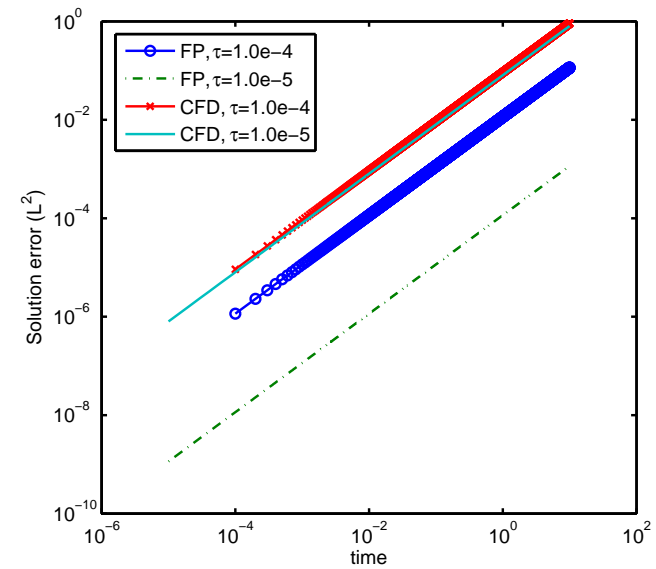

(a) The solution error in $L^{2}$ norm

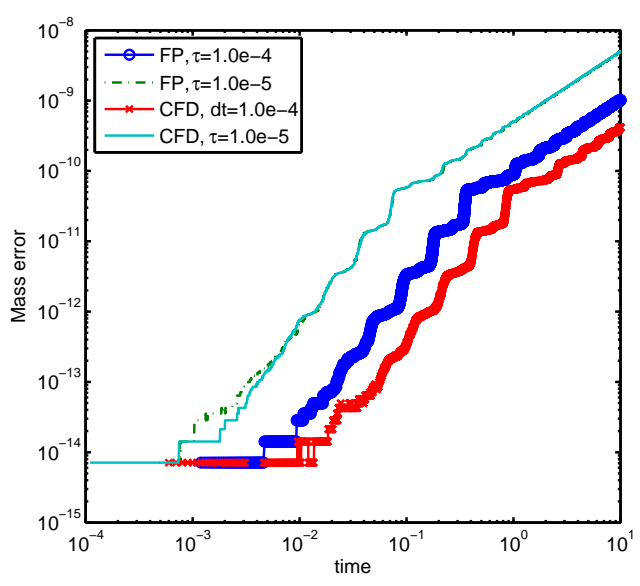

(c) Mass error

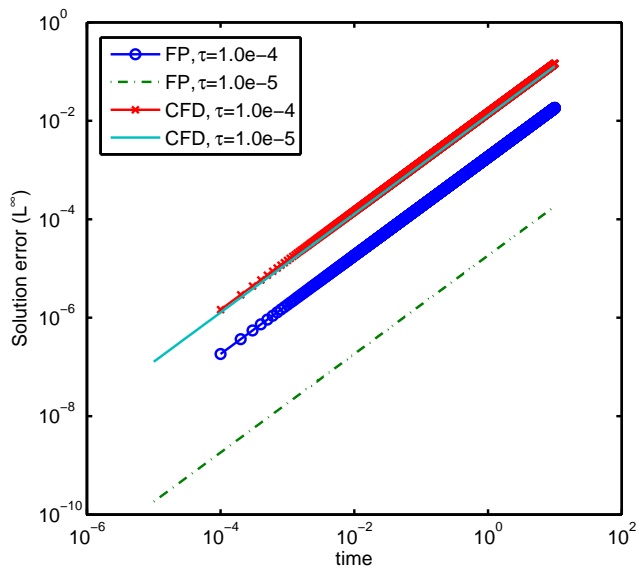

(b) The solution error in $L^{\infty}$ norm

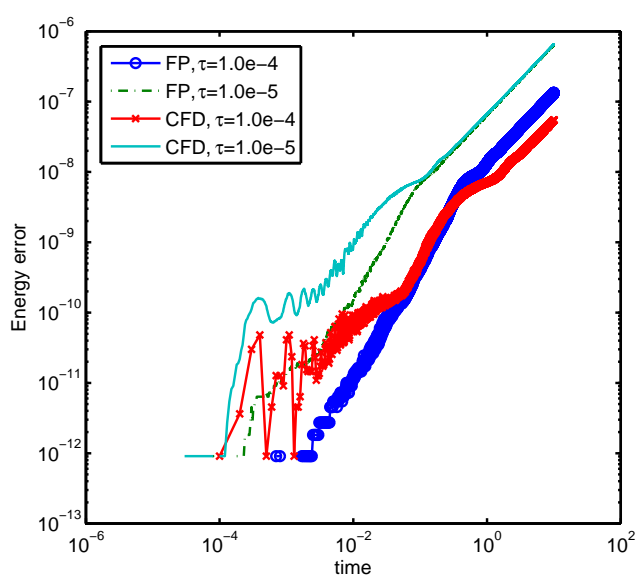

(d) Energy error

Figure 2: Error Comparison between the FP and the CFD method $\left(\beta=-2, A=1, k_{1}=k_{2}=8, N_{x}=\right.$ $\left.N_{y}=128\right)$.

Example 5.2. In this example, we show a singular solution for the 2D NLS equation

$$
i u_{t}+\Delta u+|u|^{2} u=0
$$

with initial condition

$$
u(x, y, 0)=(1+\sin x)(2+\sin y),(x, y) \in[0,2 \pi] \times[0,2 \pi],
$$

and the $(2 \pi, 2 \pi)$-periodic boundary condition. Fig. 3 shows the singular solution, which matches the result in [58] very well. The errors in mass and energy are plotted in Fig. 4, which are conserved very well. 

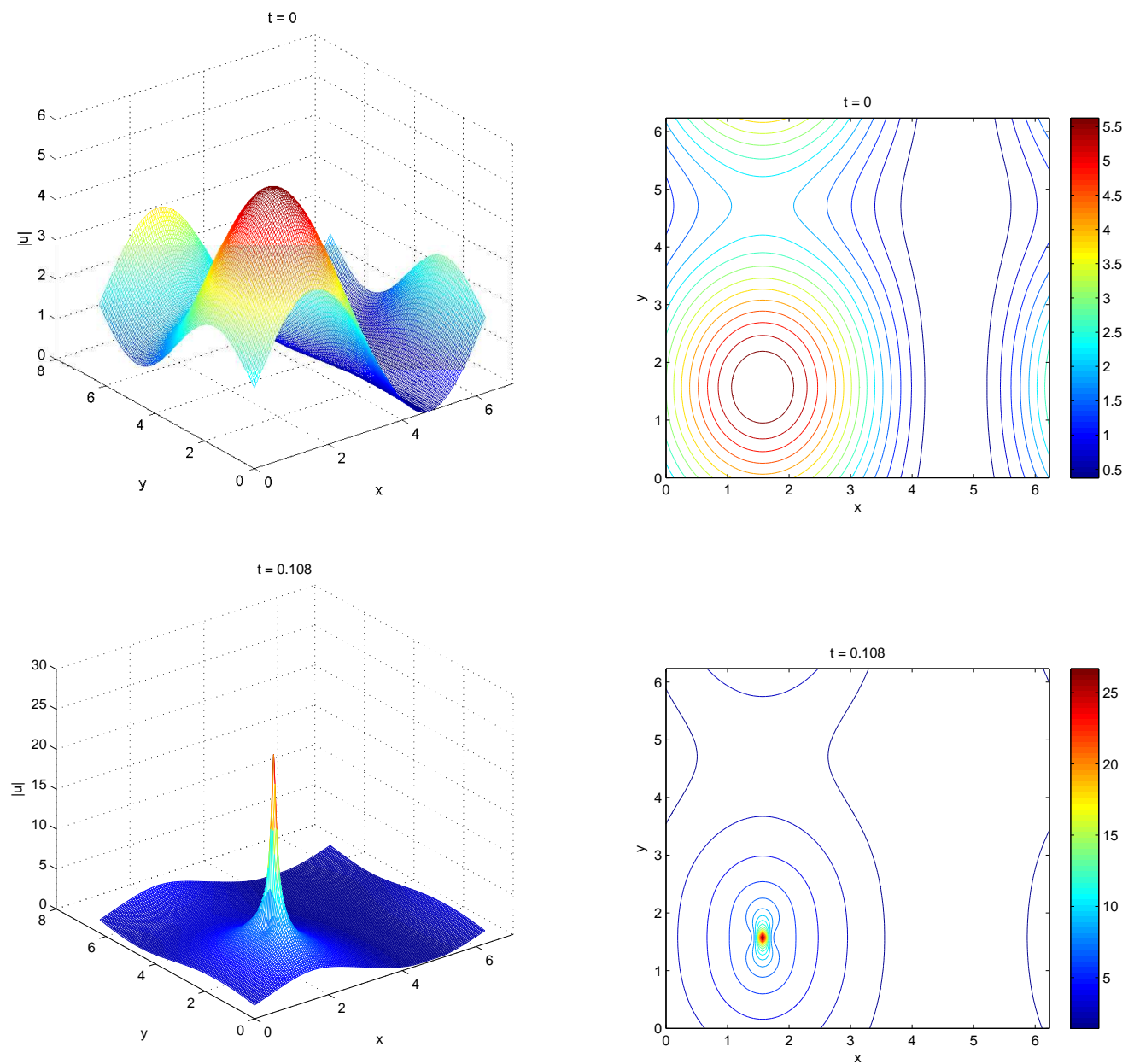

Figure 3: Profiles and contours of modulus of initial data (top) and the singular solution, where the singularity develops at $t=0.108$ (bottom) with $N_{x}=N_{y}=128, \tau=0.0001$.
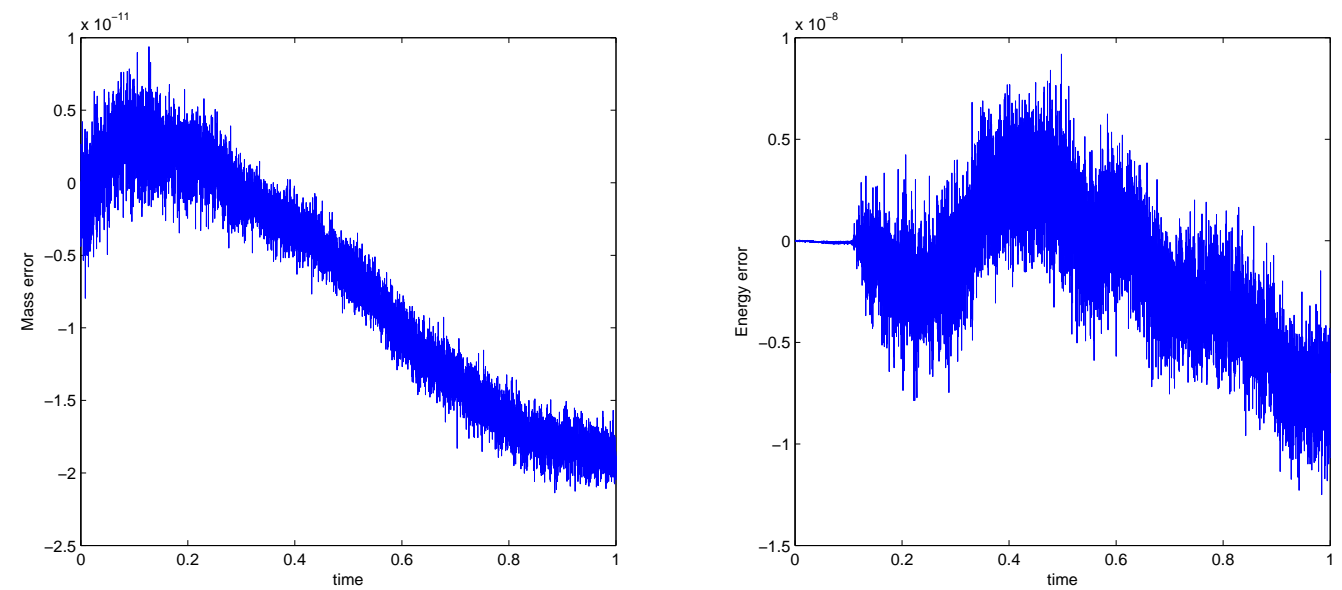

Figure 4: The error of the numerical singular solution in mass (left) and in energy (right). 


\section{Conclusions}

In this paper, we have developed a high order numerical method for solving the 2D NLS equation, where the Crank-Nicolson method in time and Fourier pseudo-spectral method in space discretization is implemented. We show that it conserves mass and energy at the discrete level, consistent with the continuum theory. We first establish the fact that the semi-norm in the Fourier pseudo-spectral method is equivalent to that in the finite difference method. This enables us to apply the techniques developed in [56] to analyze the Fourier pseudo-spectral method. The pseudo-spectral method is shown to be convergent in the order of $O\left(N^{-r}+\tau^{2}\right)$ in the discrete $L^{2}$ norm. However, the proposed method is implicit and nonlinear. To obtain the solution

$U^{n+1}$ at time level $n+1$, a nonlinear iteration for $U^{n+1}$ need to be done. A simple fixed-point iteration method is employed to solve the nonlinear equation systems defined in scheme (2.5), where the FFT is used to speed up the computation. Two numerical examples are presented to illustrate the efficiency and accuracy of the new scheme in the end, where it shows that the pseudo-spectral method is much better than the CFD method in accuracy.

\section{Acknowledgments}

Yuezheng Gong's work is partially supported by China Postdoctoral Science Foundation through Grants 2016M591054. Qi Wang's work is partially supported by National Science Foundation through grants DMS-1200487 and DMS-1517347, AFOSR grant FA9550-12-1-0178, and SC EPSCOR GEAR awards. Yushun Wang's work is supported by the Jiangsu Collaborative Innovation Center for Climate Change, the National Natural Science Foundation of China (Grant Nos. 11271195, 41231173) and the Priority Academic Program Development of Jiangsu Higher Education Institutions. Jiaxiang Cai's work is supported by NNSF of China (No. 11201169) and the foundation of Jiangsu Key Laboratory for Numerical Simulation of Large Scale Complex Systems (201606).

\section{References}

[1] J. Argyris and M. Haase, An engineer's guide to soliton phenomena: application of the finite element method, Comput. Methods Appl. Mech. Eng. 61 (1987), 71-122.

[2] G.D. Akrivis, V.A. Dougalis and O.A. Karakashian, On fully discrete Galerkin methods of second-order temporal accuracy for the nonlinear Schrödinger equation, Numer. Math. 59 (1991), 31-53.

[3] G.D. Akrivis, Finite difference discretization of the cubic Schrödinger equation, IMA J. Numer. Anal. 13 (1993), 115-124.

[4] W. Bao, S. Jin and P.A. Markowich, On time-splitting spectral approximation for the Schrödinger equation in the semiclassical regime, J. Comput. Phys. 175 (2002), 487-524.

[5] W. Bao, D. Jaksch and P.A. Markowich, Numerical solution of the Gross-Pitaevskii equation for Bose-Einstein condensation, J. Comput. Phys. 187 (2003), 318-342.

[6] W. Bao and J. Shen, A fourth-order time-splitting Laguerre-Hermite pseudospectral method for Bose-Einstein condensates, SIAM J. Sci. Comput. 26 (2005), 2010-2028.

[7] W. Bao, Q. Du and Y. Zhang, Dynamics of rotating Bose-Einstein condensates and its efficient and accurate numerical computation, SIAM J. Appl. Math. 66 (2006), 758-786. 
[8] W. Bao, H. Li and J. Shen, A generalized-Laguerre-Fourier-Hermite pseudospectral method for computing the dynamics of rotating Bose-Einstein condensates, SIAM J. Sci. Comput. 31 (2009), 3685-3711.

[9] C. Besse, B. Bidegaray and S. Descombes, Order estimates in time of splitting methods for the nonlinear Schrödinger equation, SIAM J. Numer. Anal. 40 (2002), 26-40.

[10] T.J. Bridges and S. Reich, Multi-symplectic spectral discretizations for the ZakharovKuznetsov and shallow water equations, Phys. D 152-153 (2001), 491-504.

[11] F.E. Browder, Existence and uniqueness theorems for solutions of nonlinear boundary value problems, In: Application of Nonlinear Partial Differential Equations, Proceedings of symposia in Applied Mathematics, R. Finn (Ed.), AMS, Providence, 17 (1965), 24-49.

[12] J.X. Cai and Y.S. Wang, A conservative Fourier pseudospectral algorithm for a coupled nonlinear Schrödinger system, Chin. Phys. B 22 (2013), 060207.

[13] J.X. Cai, J.L. Hong, Y.S. Wang and Y.Z. Gong, Two Energy-Conserved Splitting Methods for Three-Dimensional Time-Domain Maxwell's Equations and the Convergence Analysis, SIAM J. Numer. Anal. 53 (2015), 1918-1940.

[14] J.X. Cai, Y.S. Wang and Y.Z. Gong, Numerical Analysis of AVF Methods for ThreeDimensional Time-Domain Maxwell's Equations, J. Sci. Comput. (2015), 1-36.

[15] J.X. Cai, Y.S. Wang and Y.Z. Gong, Convergence of time-splitting energy-conserved symplectic schemes for 3D Maxwell's equations, Appl. Math. Comput. 265 (2015), 51-67.

[16] C. Canuto and A. Quarteroni, Approximation Results for Orthogonal Polynomials in Sobolev Spaces, Math. Comput. 38 (1982), 67-86.

[17] B.M. Caradoc-Davis, R.J. Ballagh and K. Burnett, Coherent dynamics of vortex formation in trapped Bose-Einstein condensates, Phys. Rev. Lett. 83 (1999), 895-898.

[18] T. Cazenave, Semilinear Schrödinger equations, Courant Lecture Notes in Mathematics, vol. 10, New York University, Courant Institute of Mathematical Sciences, AMS, 2003.

[19] Q. Chang, E. Jia and W. Sun, Difference schemes for solving the generalized nonlinear Schrödinger equation, J. Comput. Phys. 148 (1999), 397-415.

[20] J.B. Chen and M.Z. Qin, Multi-symplectic Fourier pseudospectral method for the nonlinear Schrödinger equation, Electr. Trans. Numer. Anal. 12 (2001), 193-204.

[21] Y.M. Chen, S.H. Song and H.J. Zhu, The multi-symplectic Fourier pseudospectral method for solving two-dimensional Hamiltonian PDEs, J. Comput. Appl. Math. 236 (2011), 13541369 .

[22] A. Debussche and E. Faou, Modified energy for split-step methods applied to the linear Schrödinger equations, SIAM J. Numer. Anal. 47 (2009), 3705-3719.

[23] M. Dehghan, Finite difference procedures for solving a problem arising in modeling and design of certain optoelectronic devices, Math. Comput. Simulat. 71 (2006), 16-30.

[24] M. Dehghan and A. Shokri, A numerical method for two-dimensional Schrödinger equation using collocation and radial basis functions, Comput. Math. Appl. 54 (2007), 136-146. 
[25] M. Dehghan and D. Mirzaei, Numerical solution to the unsteady two-dimensional Schrödinger equation using meshless local boundary integral equation method, Int. J. Numer. Methods Eng. 76 (2008), 501-520.

[26] M. Dehghan and D. Mirzaei, The meshless local Petrov-Galerkin (MLPG) method for the generalized two-dimensional non-linear Schrödinger equation, Eng. Anal. Boundary Elem. 32 (2008), 747-756.

[27] A. Mohebbi and M. Dehghan, The use of compact boundary value method for the solution of two-dimensional Schrödinger equation, J. Comput. Appl. Math. 225 (2009), 124-134.

[28] M. Dehghan and A. Taleei, A compact split-step finite difference method for solving the nonlinear Schrödinger equations with constant and variable coefficients, Comput. Phys. Commun. 181 (2010) 43-51.

[29] M. Dehghan and A. Taleei, A Chebyshev pseudospectral multidomain method for the soliton solution of coupled nonlinear Schrödinger equations, Comput. Phys. Commun. 182 (2011), 2519-2529.

[30] M. Dehghan and F. Emami-Naeini, The Sinc-collocation and Sinc-Galerkin methods for solving the two-dimensional Schrödinger equation with nonhomogeneous boundary conditions, Appl. Math. Model. 37 (2013), 9379-9397.

[31] Z. Gao and S. Xie, Fourth-order alternating direction implicit compact finite difference schemes for two-dimensional Schrödinger equations, Appl. Numer. Math. 61 (2011), 593614.

[32] L.R.T. Gardner, G.A. Gardner, S.I. Zaki and Z. El Sahrawi, B-spline finite element studies of the non-linear Schrödinger equation, Comput. Methods Appl. Mech. Eng. 108 (1993), 303-318.

[33] R.T. Glassey, On the blowing up of solutions to the Cauchy problem for nonlinear Schrödinger equations, J. Math. Phys. 18 (1977), 1794-1797.

[34] Y.Z. Gong, J.X. Cai and Y.S. Wang, Multi-symplectic Fourier pseudospectral method for the Kawahara equation, Commun. Comput. Phys. 16 (2014), 35-55.

[35] Y.Z. Gong, J.X. Cai and Y.S. Wang, Some new structure-preserving algorithms for general multi-symplectic formulations of Hamiltonian PDEs, J. Comput. Phys. 279 (2014), 80-102.

[36] R.M. Gray, Toeplitz and circulant matrices. ISL, Tech. Rep., Stanford Univ., Stanford, CA, Aug 2002. [Online]. Available: <http://ee-www.stanford.edu/gray/toeplitz.html>

[37] D.J. Griffiths, Introduction to Quantum Mechanics, Prentice Hall, New Jersey, 1995.

[38] A. Hasegawa, Optical solitons in fibers, Springer, Berlin, 1989.

[39] O. Karakashian, G. Akrivis and V. Dougalis, On optimal order error estimates for the nonlinear Schrödinger equation, SIAM J. Numer. Anal. 30 (1993), 377-400.

[40] O. Karakashian and C. Makridakis, A space-time finite element method for the nonlinear Schrödinger equation: the discontinuous Galerkin method, Math. Comput. 67 (1998), 479499. 
[41] L.H. Kong, J.J. Zhang, Y. Cao, Y.L. Duan and H. Huang, Semi-explicit symplectic partitioned Runge-Kutta Fourier pseudo-spectral scheme for Klein-Gordon-Schrödinger equations, Comput. Phys. Commun. 181 (2010), 1369-1377.

[42] C. Lubich, On splitting methods for Schrödinger-Poisson and cubic nonlinear Schrödinger equations, Math. Comput. 77 (2008), 2141-2153.

[43] P.A. Markowich, P. Pietra and C. Pohl, Numerical approximation of quadratic observables of Schrödinger-type equations in the semi-classical limit, Numer. Math. 81 (1999), 595-630.

[44] C.R. Menyuk, Stability of solitons in birefringent optical fibers. I: Equal propagation amplitudes, Opt. Lett. 12 (1987), 614-616.

[45] C.R. Menyuk, Stability of solitons in birefringent optical fibers. II. Arbitrary amplitudes, J. Opt. Soc. Am. B 5 (1988), 392-402.

[46] C. Neuhauser and M. Thalhammer, On the convergence of splitting methods for linear evolutionary Schrödinger equations involving an unbounded potential, BIT Numer. Math. 49 (2009), 199-215.

[47] K. Ohannes and M. Charalambos, A space-time finite element method for the nonlinear Schrödinger equation: the continuous Galerkin method, SIAM J. Numer. Anal. 36 (1999), 1779-1807.

[48] D. Pathria and J.L. Morris, Pseudo-spectral solution of nonlinear Schrödinger equations, J. Comput. Phys. 87 (1990), 108-125.

[49] J. Shen and T. Tang, Spectral and high-order methods with applications, Science Press, 2006.

[50] M. Subasi, On the finite difference schemes for the numerical solution of two dimensional Schrödinger equation, Numer. Methods Partial Differ. Equ. 18 (2002), 752-758.

[51] C. Sulem and P.L. Sulem, The nonliner Schrödinger equation: self-focusing and wave collapse, Springer, New York, 1999.

[52] Z.Z. Sun and D.D. Zhao, On the $L_{\infty}$ convergence of a difference scheme for coupled nonlinear Schrödinger equations, Comput. Math. Appl. 59 (2010), 3286-3300.

[53] M. Thalhammer, High-order exponential operator splitting methods for timedependent Schrödinger equations, SIAM J. Numer. Anal. 46 (2008), 2022-2038.

[54] M. Thalhammer, M. Caliari and C. Neuhauser, High-order time-splitting Hermite and Fourier spectral methods, J. Comput. Phys. 228 (2009), 822-832.

[55] T.C. Wang and B.L. Guo, Unconditional convergence of two conservative compact difference schemes for non-linear Schrödinger equation in one dimension (in Chinese), Sci. Sin. Math. 41 (2011), 207-233.

[56] T.C. Wang, B.L. Guo and Q.B. Xu, Fourth-order compact and energy conservative difference schemes for the nonlinear Schrödinger equation in two dimensions, J. Comput. Phys. 243 (2013), 382-399.

[57] Y.S. Wang, Q.H. Li and Y.Z. Song, Two new simple multisymplectic schemes for the nonlinear Schrödinger equation, Chin. Phys. Lett. 25 (2008), 1538-1540. 
[58] Y. Xu and C.W. Shu, Local discontinuous Galerkin methods for nonlinear Schrödinger equations, J. Comput. Phys. 205 (2005), 72-97.

[59] V.E. Zakharov and V.S. Synakh, The nature of self-focusing singularity, Sov. Phys. JETP 41 (1975), 465-468.

[60] F. Zhang, V.M. Pérez-Grarciz and L. Vázquez, Numerical simulation of nonlinear Schrödinger systems: a new conservative scheme, Appl. Math. Comput. 71 (1995), 165177.

[61] Y.L. Zhou, Applications of Discrete Functional Analysis to the Finite Difference Method, Beijing: International Academic Publishers, 1990. 